\title{
Surgical Treatment of Septic Jugular Thrombophlebitis in Nine Horses
}

\author{
Tom M. Russell ${ }^{1}$, BVMS, MACVSc, Diplomate ECVS, Clodagh Kearney ${ }^{2}$, MVB, and Patrick J. Pollock ${ }^{3}$, \\ BVMS, CertES (Soft Tissue), Diplomate ECVS \\ ${ }^{1}$ Goulburn Valley Equine Hospital, Congupna, Vic., Australia, ${ }^{2}$ School of Agriculture, Food Science and Veterinary Medicine, University College Dublin, \\ Dublin, Ireland and ${ }^{3}$ Weipers Centre for Equine Welfare, Division of Companion Animal Sciences, Institute of Comparative Medicine, Faculty of \\ Veterinary Medicine, University of Glasgow, Glasgow, Scotland
}

\author{
Corresponding author \\ Patrick J. Pollock, BVMS, CertES (Soft \\ Tissue), Diplomate ECVS, Weipers Centre \\ for Equine Welfare, Division of Companion \\ Animal Sciences, Institute of Comparative \\ Medicine, Faculty of Veterinary Medicine, \\ University of Glasgow, 464 Bearsden Road \\ Glasgow G61 10H, Scotland \\ E-mail: p.pollock@vet.gla.ac.uk
}

Submitted December 2008

Accepted June 2009

DOI:10.1111/j.1532-950X.2010.00683.x
Objective: To report a technique for surgical treatment of septic jugular thrombophlebitis unresponsive to medical treatment.

Study Design: Case series.

Animals: Horses $(\mathrm{n}=9)$ with septic jugular thrombophlebitis unresponsive to medical treatment.

Methods: Jugular vein thrombectomy was performed under standing sedation and local anesthesia. The contents of the affected portion of vein were removed by multiple incisions in the vein, with the incisions left open to drain and heal by second intention.

Results: The technique was curative in all instances, although 2 horses required a 2nd procedure. One horse required ligation of the linguofacial vein to control postsurgical hemorrhage.

Conclusions: The technique is an effective surgical treatment for septic jugular thrombophlebitis unresponsive to medical treatment.

Clinical Relevance: Jugular vein thrombectomy is a straightforward technique, and has minimal postoperative complications. It allows expedient and cost-effective resolution of medically recalcitrant cases of septic jugular thrombophlebitis.
Jugular thrombophlebitis is commonly encountered in equine practice. It occurs almost exclusively at the site of intravenous (IV) catheter placement, where the catheter acts as a focus for thrombosis. ${ }^{1}$

Clinical signs include thickening of the vein, perivenous and facial edema, discharging sinus tracts, and dilation of facial veins on the affected side. Potentially more serious complications include nasal passage edema, endocarditis, and laryngeal hemiplegia. ${ }^{2}$ Most cases respond to local treatment including hot-packing, and/or topical DMSO. In some cases, systemic nonsteroidal antiinflammatory drugs (phenylbutazone, aspirin) may be of value. Where sepsis is suspected, systemic antibiosis is indicated, and should be based on culture and susceptibility testing of the septic contents of the vein lumen. In a study of shortterm complications of colic surgery, jugular thrombophlebitis occurred in $8.3 \%$ of horses, although only $0.4 \%$ of thrombophlebitis was considered septic. ${ }^{3}$ In refractive cases, surgical excision of the vein has been recommended; however, the surgery is technically demanding. ${ }^{4}$

We hypothesized that when jugular vein thrombophlebitis was refractive to medical treatment, an abscess would form with a cavity that could be drained and lavaged to achieve resolution similar to a subcutaneous abscess. Our objective was to develop a simple technique for removal of septic jugular vein contents, and to document any complications encountered.

\section{MATERIALS AND METHODS}

Between 1997 and 2007, 9 horses were admitted with septic jugular vein thrombophlebitis unresponsive to conservative therapy. Horses were treated by thrombectomy with drainage and subsequent daily lavage of the affected vein lumen. Medical records were examined to obtain history, clinical findings, intra- and postoperative complications, and outcome. Long-term outcome was determined by telephone contact with the owners and referring veterinarians.

\section{Case Selection}

Horses selected for the procedure had septic jugular thrombophlebitis based on clinical signs and percutaneous aspiration and microbial culture of lumen contents. All horses had one or more extended course(s) of antibiosis that failed to resolve the clinical signs. 


\section{Presurgical Evaluation}

Before surgery, all horses had rhinolaryngoscopy to document laryngeal function. Color-flow Doppler ultrasonography of the affected vein was used to rule out vessel patency or blood flow, and to delineate the cranial and caudal extent of the thrombus, fluid-filled cavities within the vein. Cavities were invariably associated with the sites of greatest pain and swelling in the vein, and were used to help in positioning of incisions. Echocardiographic evaluation was used to determine if there was evidence of endocarditis.

Perioperatively, horses were administered phenylbutazone $(4.4 \mathrm{mg} / \mathrm{kg})$ and trimethoprim-sulfonamide (trimethoprim $54 \mathrm{mg} / \mathrm{kg}$, sulfadiazine $27 \mathrm{mg} / \mathrm{kg}$ ) orally.

\section{Surgical Procedure}

Horses were restrained in stocks and sedated with xylazine hydrochloride $(1 \mathrm{mg} / \mathrm{kg}$ IV) and butorphanol tartrate $(0.02 \mathrm{mg} / \mathrm{kg}$ IV). The head was cross-tied and held by an assistant to prevent the neck from resting on the front bar of the stocks leading to potential contamination of the surgical site. The surgical site was clipped and prepared for surgery and a single drape taped along a line parallel to, and just ventral to the mane, and draped over the contralateral side of the horse to remove the mane from the surgical field.

Anesthesia of the region was achieved by infiltration of $2 \%$ mepivacaine hydrochloride solution in an inverted L-shaped pattern in the skin and subcutaneous tissue. The length of the horizontal part of the $\mathrm{L}$ was determined based on the ultrasonographic extent of the affected portion of the vein. A $5 \mathrm{~cm}$ skin incision parallel to the jugular vein was made directly over the midpoint of the affected area identified ultrasonographically. Tissue dissection was continued into the lumen of the affected portion with an assistant retracting the edges. A Ferris-Smith rongeur was introduced into the vein lumen cranially and caudally to remove the infected clot material (Fig 1). A 2nd incision was created similarly, but in a more caudal position overlying the vein. Ferris-Smith rongeurs were again introduced; however, care was taken only to introduce the rongeurs cranially to avoid creation of a dependent nondraining area in the caudal portion of the affected vein.

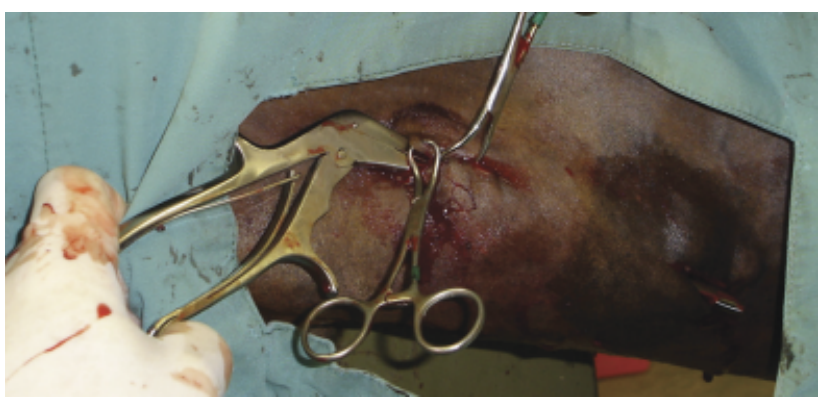

Figure 1 Ferris-Smith rongeurs inserted into the lumen of the left jugular vein.

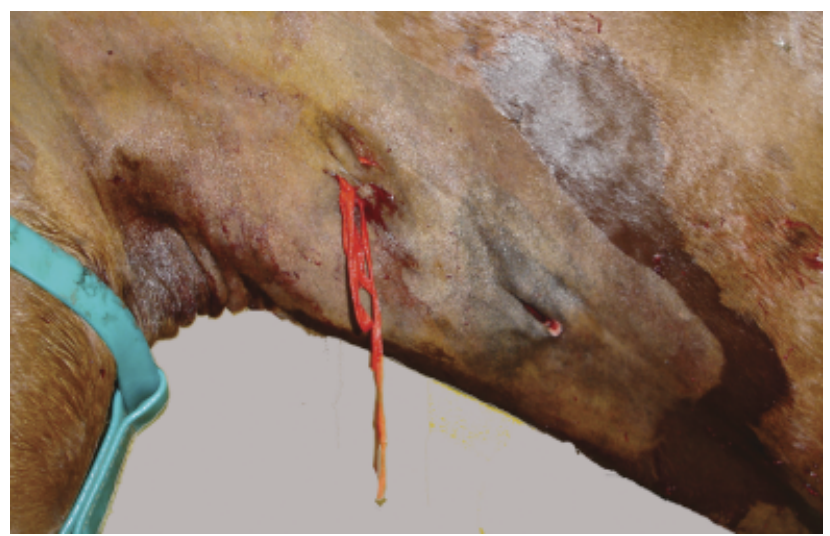

Figure 2 The cranial-most incision closed in a bow using umbilical tape.

A 3rd incision was created if the length of the affected portion of the vein could not be reached through 2 portals.

After removing the luminal content, the lumen was flushed with $500 \mathrm{~mL} 1 \%$ povidone-iodine, using a $60 \mathrm{~mL}$ syringe. A Penrose drain was placed through the vein lumen to prevent premature closure of the surgical sites. This was achieved by feeding the drain through 1 incision with Crille forceps and grasping it through the 2 nd incision with the Ferris-Smith rongeurs. In the last 5 horses, a rolled gauze swab was inserted cranially into the vein from the cranial most incision and removed 24-48 hours later. The incision was closed with umbilical tape (horizontal mattress pattern) that was then tied with a bow to allow easy daily opening (Fig 2).

\section{Postoperative Treatment}

Horses were administered oral phenylbutazone $(2.2 \mathrm{mg} /$ $\mathrm{kg}$ /day) until drain removal and oral trimethoprimsulfadiazine ( $54 \mathrm{mg} / \mathrm{kg}$ trimethoprim $/ 27 \mathrm{mg} / \mathrm{kg}$ sulfadiazine twice daily) until wound healing was complete. Daily lavage with $1 \%$ povidone-iodine was continued until drain removal, typically when discharge was considered to have abated sufficiently.

\section{Outcome}

Telephone contact was made with owners and the referring veterinarians at $\geq 6$ months after surgery to determine outcome and long-term complications.

\section{RESULTS}

Nine horses ( 2 females, 6 geldings, and 1 stallion) aged 2-14 years (median, 4 years) had surgery to address septic jugular thrombophlebitis unresponsive to medical treatment. There were 4 Thoroughbred, 3 Warmblood, 1 Standardbred, and 1 pony. All horses had left jugular vein involvement and were admitted with intermittent pyrexia, perivascular swelling, and pain; 2 horses had severe leftsided facial swelling. 
For the 8 horses with an adequate clinical history on admission, 5 had a jugular catheter inserted for $<4$ hours for elective surgery ( 3 middle carpal joint arthroscopy, 1 tooth removal, and 1 foot cast). Catheters were all short stay $12.5 \mathrm{~cm} \times 14 \mathrm{~g}$ composed of polytetrafluoroethylene (PTFE). All 5 horses had been anesthetized with xylazine, ketamine, and guaiafenesin, and maintained on halothane in oxygen. Two horses had jugular vein catheterization for $>24$ hours for IV fluid administration (1 medical colic, 1 colitis); catheters were flushed with heparinized saline solution once daily. One horse had been administered vitamin B complex IV 2 months before admission. Time from initial venipuncture until surgical intervention ranged from 7 to 16 weeks (median, 9 weeks).

All horses had been administered an extended course ( $>3$ weeks) of antibiotics based on microbial susceptibility patterns, when available. For 6 horses, oral trimethoprim-sulfonamide was initially administered followed by a course of intramuscular penicillin. One horse had intramuscular gentamicin followed by a course of intramuscular penicillin. One horse was administered a course of intramuscular neomycin. In all horses, adjunctive warm toweling and topical DMSO was applied locally for 3-5 days when initial perivenous swelling became apparent.

Bacteriology results were available for 7 horses. Isolates included Escherichia coli (3), Streptococcus zooepidemicus (2), and Actinobacillus equuili (2). One horse had a mixed infection of $E$. coli and $S$. zooepidemicus. Two horses did not have samples taken for microbial culture and were treated based on clinical signs, and transient resolution of signs while being treated with antibiotics.

Eight surgical procedures were completed without incident, and material similar in appearance and texture to fibrinous clot was removed. In 1 horse, surgery was uneventful, but on returning to its box and being fed, the horse began to leak jets of blood from the cranial incision coincident with closure of the jaws. Simply stopping the horse from eating abated the flow significantly; however, because leakage was coming only from the linguofacial vein, this was ligated with 2-0 polyglactin 910 through a $2 \mathrm{~cm}$ incision just axial to where the vein crosses the ramus of the mandible. The incision was closed using a simple interrupted skin pattern. This event prompted use of a gauze plug in the cranial incision, in subsequent horses, as described earlier.

Two horses required a 2 nd surgery, one 3 weeks and one 3 months, after the initial surgery. Ultrasonographic and clinical examination demonstrated evidence of recurrence of sepsis at the previous surgical site, and repeat surgery was carried out in the same fashion as the first. One horse had a wound that discharged copious purulent material for several weeks after surgery. All other wounds had effectively sealed within 3 days of drain removal.

All horses had resolution of clinical signs and had returned to full use at follow-up at 6 months. Swelling disappeared completely in the 2 horses with preoperative facial swelling. All veins remained grossly enlarged and fibrotic.
Ultrasonographic determination of jugular vein patency was not performed.

\section{DISCUSSION}

Standing jugular phlebotomy is a simple and effective method for resolving septic jugular thrombophlebitis in horses. Another technique ${ }^{4}$ involves resection of the affected portion of the jugular vein and requires general anesthesia, invasive dissection, loss of attendant vagosympathetic trunk, and carries a high risk of wound dehiscence. Whereas our technique obviates these problems, we did record some complications, all of which we believe were because of addressable technical errors.

We believe the horse that bled from the linguofacial vein did so because of overly aggressive clot removal. The clot can be expected to extend significantly up- and downstream from the infected material and therefore we recommend that clot removal cease well short of its cranial and caudal extremities. Use of a gauze swab plug in the cranial lumen of the vein gives added security against postoperative hemorrhage.

The 2 horses that required a 2 nd surgery both had reoccurrence at the previous surgical site. We believe this occurred because of premature wound closure and inadequate drainage. Where doubt exists, we recommend leaving the drain in place for a longer, rather than a shorter time. There is some possibility that intraoperative ultrasonography may have identified pockets of septic material that were not removed. Although not done in our horses, we suggest that intraoperative ultrasound evaluation may be helpful and have since used it in similar cases.

In the 1st horse treated with this technique, the wound discharged copiously for several weeks. This may have been because of the bacterium involved ( $A$. equili) but more likely was from overly aggressive curettage of the walls of the vein. After aggressive removal of vein wall tissue, the left side of the neck developed severe cellulitis, presumably because of extension of the infection. Subsequently, the technique was modified so that only material within the vein was removed and this complication has not been encountered again.

One of the most important problems encountered when treating septic jugular thrombophlebitis medically is that most effective antimicrobials must be administered intravenously. This involves either taking the risk of damage to the contralateral jugular vein, which may be at increased risk of thrombosis, or placing and maintaining a catheter in another peripheral vein. All of the bacteria cultured from veins in these horses were susceptible to trimethoprimsulfonamide, but as is typical of most abscesses, medical treatment alone was insufficient to resolve signs, and surgical drainage was necessary.

For case selection and timing of surgical intervention, we tended toward earlier surgical intervention as we gained familiarity with the technique. Most cases were considered 
iatrogenic and there was some pressure from owners to resolve the problem in an expedient and inexpensive fashion.

Other techniques reported for treatment of septic thrombophlebitis refractory to medical treatment include balloon thrombectomy, and a variety of techniques for replacing affected portions of the jugular with a graft from another vessel including the saphenous vein..$^{5-7}$

Mair and $\mathrm{Smith}^{3}$ reported that only $0.4 \%$ of surgical colics develop septic jugular thrombophlebitis. Our experience is similar and it should be noted that the cases we report were encountered over 10 years. Whereas the technique is simple and effective, we recommend that all avenues of medical treatment be exhausted first. While it is certainly possible that longer term antibiotic use may have resolved some or all of the cases presented here, for reasons already mentioned, surgical intervention was considered a time and cost-effective measure.

Risk factors for catheter-associated jugular thrombophlebitis include the catheter material, large intestinal disease, hypoproteinemia, salmonellosis, and endotoxemia. ${ }^{8}$ An earlier report ${ }^{9}$ suggested that injection of irritant chemicals such as guaiafenesin and thiopentone were linked to development of thrombophlebitis. Our case numbers are too small for statistical inference; however, it is interesting to note that $5 / 9$ horses were administered guaiafenesin. French et $\mathrm{al}^{10}$ found no evidence that guaiafenesin was a risk factor. Of the other 3 horses, we suspect that constant uncontrollable rolling of the 2 colicky horses led to catheter site contamination. In the other horse, vitamin $B_{1}$ is an irritant substance that may have caused an initial thrombus, although why this would then become septic is impossible to determine.

Various materials have been used for catheter production including in likely order of decreasing thrombogenicity: polypropylene, polyethylene, PTFE, silicone rubber, polyvinylchloride, and polyurethane. ${ }^{11}$ Material used can also have an effect on bacterial colonization with several studies reporting increased bacterial colonization rate in materials associated with higher rates of thrombus formation. ${ }^{12}$

Unfortunately, for logistical reasons, ultrasonographic evaluation of affected jugular patency was not determined in any of these horses. Should patency be re-established, this would make the technique more attractive, and this aspect of the technique warrants further investigation. We recommend this technique for resolution of septic jugular thrombophlebitis unresponsive to medical treatment.

\section{ACKNOWLEDGMENTS}

We gratefully acknowledge the assistance of Ms. Sarah O'Dwyer, Mr. David Mason, Mr. Phil Brady, Mr. Peter Urban, and the veterinary surgeons who referred the cases.

\section{REFERENCES}

1. Morris DD: Thrombophlebitis in horses: the contribution of haemostatic dysfunction to pathogenesis. Compend Contin Educ Prac Vet 1991;11:1386-1394

2. Gilbert GH: Laryngeal hemiplegia following jugular injury. $J$ Am Vet Med Assoc 1972;161:1686-1687

3. Mair TS, Smith LJ: Survival and complication rates in 300 horses undergoing surgical treatment of colic. Part 2: shortterm complications. Equine Vet J 2005;37:305-309

4. Gardner SY, Donawick WJ: Jugular vein thrombophlebitis, in Robinson NE (ed): Current Therapy in Equine Medicine (ed 3). Philadelphia, W. B. Saunders, 1992, pp 406-408

5. Rikjenhuizen AB, van Swieten HA: Reconstruction of the jugular vein in horses with post thrombophlebitis stenosis using saphenous graft. Equine Vet $J$ 1998;30:236-239

6. Weimer P, Gruys E, van Hoeck B: A study of seven different types of grafts for jugular vein transplantation in the horse. Res Vet Sci 2005;79:211-217

7. Hilton H, Aleman M, Textor J, et al: Ultrasound-guided balloon thrombectomy for treatment of aorto-iliac-femoral thrombosis in a horse. $J$ Vet Intern Med 2008;22:679-683

8. Dolente BA, Beech J, Lindborg S, et al: Evaluation of risk factors for development of catheter-associated jugular thrombophlebitis in horses: 50 cases (1993-1998). J Am Vet Med Assoc 2005;227:1134-1141

9. Dickson LR, Badcoe LM, Burbidge H, et al: Jugular thrombophlebitis resulting from an anaesthetic induction technique in the horse. Equine Vet J 1990;22:177-179

10. French NP, Smith J, Edwards GB, et al: Equine surgical colic: risk factors for postoperative complications. Equine Vet $J$ 2002;34:444-449

11. Spurlock SL, Spurlock GH, Parker G, et al: Long-term jugular vein catheterization in horses. J Am Vet Med Assoc 1990;196:425-430

12. Stillman RM, Soliman F, Garcia L, et al: Etiology of catheter associated sepsis. Arch Surg 1977;112:1497-1499 
Copyright of Veterinary Surgery is the property of Wiley-Blackwell and its content may not be copied or emailed to multiple sites or posted to a listserv without the copyright holder's express written permission. However, users may print, download, or email articles for individual use. 